\title{
Astigmatismo induzido na cirurgia extracapsular da catarata: acompanhamento a longo prazo
}

\author{
Induced astigmatism in extracapsularcataract extraction:long-term follow-up
}

\author{
Almir Ghiaroni ${ }^{1}$ \\ José Belmiro de Castro Moreira ${ }^{2}$
}

${ }^{1}$ Mestre em Oftalmologia pela Universidade Federal do Rio de Janeiro - UFRJ e Doutor em Oftalmologia pela Universidade Federal de São Paulo - UNIFESP.

${ }_{2}^{2}$ Professor Titular Aposentado da Disciplina de Oftalmologia da Universidade Federal de São Paulo - UNIFESP.

Endereço para correspondência: Almir Ghiaroni Rua Gal. Venâncio Flores, 305/309 - Rio de Janeiro (RJ) CEP 22441-090

E-mail: almirghiaroni@aol.com

Recebido para publicação em 06.11.2002

Versão revisada recebida em 08.05.2003

Aprovação em 09.05.2003

\begin{tabular}{|l|}
\hline RESUMO \\
\hline Objetivo: Estudar o comportamento do astigmatismo induzido a longo \\
prazo em uma série de pacientes submetidos à cirurgia extracapsular da \\
catarata, nos quais foi utilizada incisão límbica posterior superior suturada \\
com pontos separados de mononylon 10-0. Métodos: Foram estudados 38 \\
olhos, de 35 pacientes, sendo 21 do sexo feminino e 14 do sexo masculino, \\
com idades variando entre 47 e 85 anos (média = 70,17 anos). Todos os \\
casos foramoperados pelomesmo cirurgião. Operíodo de acompanhamento \\
pós-operatório variou de 15 a 33 meses (média = 22,07 meses). Nenhum \\
dos casos teve qualquer dos pontos seccionado ou removido. Todos os \\
pacientes atingiram acuidade visual corrigidaigual ou superior a 0,5. Para \\
o cálculo do astigmatismo induzido foram consideradas as medidas \\
ceratométricas pré e pós-operatórias e foi utilizado um programa de- \\
senvolvidopor Jaffe. Resultados: O astigmatismo induzido variou de 0,13 \\
dioptria(D) a 2,74D (média=1,19D). Em relação aos eixos, na maioria dos \\
casos houve indução de astigmatismo a favor da regra (60,52\%). Foi \\
observada mudança significativa no valor dióptrico do astigmatismo \\
quando foram comparadas as leituras ceratométricas registradas pré- \\
operatoriamente até a segunda semana pós-operatória, entre o primeiro e \\
o terceiro meses pós-operatórios, entre o terceiro e o sexto meses pós- \\
operatórios e após o décimo-segundo mês pós-operatório. Conclusão: \\
Quando o mononylon 10-0 é utilizado como material de sutura na cirurgia \\
extracapsular da catarata, os valores de astigmatismo a favor da regra \\
inicialmente observados tendem a diminuir com o tempo. \\
\hline
\end{tabular}

Descritores: Astigmatismo/etiologia; Extração de catarata; Procedimentos cirúrgicos oftalmológicos/métodos; Técnicas de sutura; Seguimentos; Adulto

\section{INTRODUÇÃ̃O}

O astigmatismo induzido na cirurgia da catarata depende, basicamente, da incisão e da sutura, que atuam sobre a cicatrização da ferida operatória ${ }^{(1-2)}$. Considerando-se estudos realizados ${ }^{(3)}$, pode-se afirmar que:

1. Incisões muito anteriores (corneanas) tendem a induzir mais astigmatismo do que incisões realizadas mais posteriormente. O eixo do astigmatismo induzido vai depender da técnica de sutura empregada.

2. Quanto menor a amplitude da incisão superior, menor efeito se verificará sobre o meridiano horizontal. Incisões que atinjam o meridiano horizontal ou o ultrapassem, tendem a neutralizar algumas das alterações que se verificam sobre o meridiano vertical.

3. A separação das bordas da incisão tem, surpreendentemente, pouco efeito na indução de astigmatismo quando a incisão é escleral, mas pode induzir um astigmatismo considerável quando a incisão é mais anterior. 
Em relação ao tamanho da incisão, afirma-se que uma incisão de $4 \mathrm{~mm}$ ou menor tem pouco efeito na indução de astigmatismo. Uma incisão pequena não apresenta grande tendência a permitir uma separação de suas bordas em virtude do suporte lateral que lhe é fornecido pelo anel límbico praticamente intacto. Além disso, é mais fácil para o cirurgião fechá-la, promovendo uma boa aposição de suas bordas, sem deixar apertados ou frouxos os pontos, o que torna bastante raro o aparecimento de um astigmatismo alto, seja a favor ou contra a regra ${ }^{(4)}$.

Incisões maiores invariavelmente induzem mais astigmatismo, em virtude de promoverem uma alteração muito maior no suporte fornecido pelo anel límbico. Esta idéia foi confirmada ao comparar-se o astigmatismo induzido em pacientes submetidos à facoemulsificação e à técnica extracapsular, respectivamente ${ }^{(5-6)}$. Em ambos os estudos, o astigmatismo induzido foi maior na série dos pacientes submetidos à técnica extracapsular.

Chama-se a atenção para o fato de que, em relação ao astigmatismo pós-operatório, a par da localização da incisão, é de extrema importância o material de sutura utilizado ${ }^{(7)}$.

Acentua-se a importância do tempo de acompanhamento pós-operatório no estudo do astigmatismo induzido na facectomia, sobretudo quando o material de sutura usado apresenta as características de grande elasticidade e de sofrer hidrólise tecidual, como é o caso do mononylon 10-0 $0^{(8)}$.

Quanto maior o número de pontos bem como o comprimento e a tensão dos mesmos, maior tende a ser o astigmatismo induzido, especialmente em se tratando de incisões anteriores ${ }^{(3)}$.

Existem várias maneiras de se realizar a sutura na cirurgia extracapsular. Pode-se empregar a sutura contínua, pontos em "X", pontos em "X" intercalados com pontos separados, a sutura horizontal de Shepherd ou apenas pontos separados ${ }^{(9-10)}$.

Neste trabalho, estudamos o comportamento do astigmatismo induzido a longo prazo em uma série de pacientes submetidos à extração extracapsular da catarata nos quais foi utilizada uma incisão límbica, realizada a $1,5 \mathrm{~mm}$ do limbo cirúrgico, fechada com pontos separados com mononylon 10-0. Os autores optaram por não retirar nenhum ponto com objetivo de poder observar a diminuição natural e progressiva dos valores ceratométricos ao longo do tempo.

\section{MÉTODOS}

Foram examinados 38 olhos de 35 pacientes, sendo 21 do sexo feminino e 14 do sexo masculino, com idades variando entre 47 e 85 anos (média $=70,17$ anos). O período de acompanhamento pós-operatório variou de 15 a 33 meses (média $=$ 22,07 meses).

Todos os casos foram operados pelo autor.

Nenhum dos casos estudados teve qualquer dos pontos seccionado ou removido.

Os casos que tiveram ponto(s) seccionados(s) ou removidos(s) não foram incluídos no presente estudo.
Todos os pacientes atingiram acuidade visual igual ou superior a 0,5 com correção.

Considerando que o objetivo do presente trabalho foi estudar o astigmatismo induzido, não foi do interesse dos autores correlacionar a acuidade visual pós-operatória dos pacientes com o astigmatismo induzido em cada caso.

Não foram incluídos no presente trabalho pacientes que apresentassem: cirurgia prévia no olho a ser operado, história ou sinais de uveíte, catarata secundária, degeneração macular, doença oclusiva vascular retiniana, diabetes, história de alergia medicamentosa, glaucoma e neoplasia.

Nenhum paciente foi excluído devido aos valores ceratométricos pré-operatórios.

Alguns pacientes apresentavam hipertensão arterial, artrite e doença vascular arteriosclerótica, mas nenhum deles se encontrava sob tratamento com antiinflamatórios ou com qualquer outro medicamento que pudesse influenciar o processo cicatricial no momento da cirurgia.

Os pacientes usuários de aspirina suspenderam o seu uso no mínimo duas semanas antes da cirurgia.

Nenhum dos casos estudados apresentou complicações per ou pós-operatórias.

A técnica cirúrgica empregada foi a extração extracapsular da catarata com implante intra-ocular de câmara posterior e constou, basicamente, de: retalho conjuntival de base no fórnix; hemostasia dos vasos límbicos e peri-límbicos através da utilização de um cautério bipolar; sulco atingindo cerca de metade da espessura da esclera localizado a $1,5 \mathrm{~mm}$ do limbo cirúrgico, realizado com lâmina de metal; abertura da câmara anterior às 10 horas, de cerca de $1,0 \mathrm{~mm}$ de comprimento, paralela ao plano da íris; capsulotomia anterior do tipo "abridor de lata", realizada com agulha com a ponta angulada, utilizando-se solução salina balanceada como líquido de irrigação; ampliação da incisão para a esquerda e para a direita com tesouras de Barraquer mantendo-se suas lâminas paralelas ao plano da íris com o intuito de obter-se uma incisão biselada. O comprimento da incisão variou de 10 a $12 \mathrm{~mm}$; extração do núcleo pela manobra de pressão e contra-pressão, tendo-se em uma das mãos um gancho de Arruga e, na outra, uma alça de Snellen; aspiração das massas corticais através da utilização de um facoemulsificador Kelman-Cavitron modelo 8000; inserção de uma lente intra-ocular de câmara posterior. Os modelos utilizados foram: Iolab 107G, Iolab G108b e Cilco SK21U; iridectomia periférica localizada às 12 horas; fechamento da incisão com mononylon 10-0 na forma de pontos separados, de cerca de $1 \mathrm{~mm}$ de comprimento, colocados ao nível da membrana de Descemet. O número de pontos, em cada caso, variou de 9 a 10.

Concluído o ato operatório, foi feita uma infiltração subconjuntival no fundo de saco inferior de $1 \mathrm{ml}$ de acetato de metil-prednisolona $40 \mathrm{mg}$ e $1 \mathrm{ml}$ de gentamicina $40 \mathrm{mg}$.

No período pós-operatório, dexametasona micronizada a $0,1 \%$, sulfato de neomicina $0,5 \%$ e sulfato de polimixina B $6000 \mathrm{U} / \mathrm{ml}$ foram utilizados topicamente durante cerca de 4 semanas e dexametasona $0,1 \%$ durante as 3 semanas seguintes, de 2 a 3 vezes ao dia. 
Os valores ceratométricos foram registrados pré-operatoriamente e no período pós-operatório, sendo que sua medida obedeceu ao seguinte critério:

Medida 1: até a segunda semana pós-operatória; medida 2: entre o primeiro e o terceiro meses pós-operatórios; medida 3: entre o terceiro e o sexto meses pós-operatórios; medida 4: do décimo-segundo mês pós-operatório em diante.

O mesmo ceratômetro, da marca Bausch-Lomb, foi utilizado para as medidas em todos os casos. O cirurgião não tinha conhecimento das medidas ceratométricas realizadas pré-operatoriamente.

Para o cálculo do astigmatismo induzido, em cada caso, foi utilizado um programa desenvolvido por Jaffe. Para a classificação do astigmatismo pós-operatório em relação aos eixos encontrados, foi utilizada a classificação proposta por Ghiaroni ${ }^{(11)}$, levando-se em conta cilindros negativos: de $0^{\circ}$ a $30^{\circ}$ e de $151^{\circ}$ a $180^{\circ}$ foram considerados a favor da regra; de $61^{\circ}$ a $120^{\circ}$ foram considerados contra a regra; de $31^{\circ}$ a $60^{\circ}$ e de $121^{\circ}$ a $150^{\circ}$ foram considerados oblíquos.

Para a avaliação estatística, foi utilizada a análise de variância por postos de Friedman ${ }^{(12)}$, com o objetivo de comparar, para cada paciente, os valores dióptricos relativos às medidas ceratométricas registradas pré-operatoriamente e com duas semanas de pós-operatório(M1), entre o primeiro e o terceiro meses pós-operatórios(M2), entre o terceiro e o sexto meses pós-operatórios(M3) e após o décimo-segundo mês pós-operatório(M4).

\section{RESULTADOS}

O valor médio do astigmatismo induzido verificado após um período pós-operatório que variou de 15 a 33 meses (média $=22,07$ meses) foi de $1,19 \mathrm{D}$, variando de 0,13 a 2,74 D.

$\mathrm{Na}$ maior parte dos casos $(47,36 \%)$, o valor dióptrico do astigmatismo induzido ficou entre 0,13 e $1 \mathrm{D}$ (Tabela 1).

Em relação aos eixos, a maioria dos pacientes $(60,52 \%)$ apresentou indução de astigmatismo a favor da regra (Tabela 2).

A análise de variância por postos de Friedman mostrou uma diferença estatisticamente significativa entre as leituras ceratométricas registradas pré-operatoriamente até a segunda semana pós-operatória, entre o primeiro e o terceiro meses pósoperatórios, entre o terceiro e o sexto meses pós-operatórios e após o décimo-segundo mês pós-operatório, evidenciando uma diminuição gradativa do astigmatismo induzido em longo prazo.

\section{DISCUSSÃO}

Em um estudo deste tipo, é extremamente importante o tempo de acompanhamento pós-operatório dos pacientes para que se possa tirar conclusões realmente válidas sobre o astigmatismo induzido. Cory ${ }^{(13)}$, ao estudar o astigmatismo induzido em pacientes submetidos à extração extracapsular da catarata nos quais foi utilizado mononylon 10-0 no fechamento da

\begin{tabular}{|lcc|}
\hline \multicolumn{3}{|c|}{$\begin{array}{c}\text { Tabela 1. Distribuição dos olhos pseudofácicos quanto ao valor } \\
\text { dióptrico do astigmatismo pós-operatório induzido }\end{array}$} \\
Valor dióptrico & $\begin{array}{c}\text { Número } \\
\text { de casos }\end{array}$ & $\%$ \\
& 18 & 47,36 \\
Até $1,00 \mathrm{D}$ & 14 & 36,84 \\
De $1,01 \mathrm{D}$ a $2,00 \mathrm{D}$ & 6 & 15,78 \\
De 2,01 D a 3,00 D & &
\end{tabular}

\begin{tabular}{|lcc|}
\hline \multicolumn{2}{|c|}{$\begin{array}{c}\text { Tabela 2. Distribuição dos olhos pseudofácicos quanto aos eixos } \\
\text { do astigmatismo pós-operatório induzido }\end{array}$} \\
Valor dióptrico & $\begin{array}{c}\text { Número } \\
\text { de casos }\end{array}$ & $\%$ \\
A favor da regra & 23 & 60,52 \\
Contra a regra & 8 & 21,05 \\
Oblíquos & 7 & 18,42 \\
\hline
\end{tabular}

incisão, tomou como base para seus cálculos os valores ceratométricos registrados após um seguimento pós-operatório de apenas 6 meses. No presente estudo, os pacientes tiveram um período de acompanhamento pós-operatório bem maior, variando de 15 a 33 meses, o que faz com que as observações verificadas tendam a ser mais confiáveis.

Em relação ao valor dióptrico médio do astigmatismo induzido no presente trabalho (1,19 D), verificou-se que o mesmo foi semelhante ao valor encontrado em uma série de 100 pacientes submetidos à facectomia extracapsular nos quais foi utilizada uma incisão corneana inversa suturada com mononylon 10-0 na forma de pontos separados (1,11 D) considerando os valores ceratométricos registrados até o sexto mês pós-operatório ${ }^{(14)}$.

Em relação ao tamanho da incisão, a literatura mostra que o mesmo pode variar até $12 \mathrm{~mm}$, a exemplo do que ocorreu no presente trabalho ${ }^{(11,15-18)}$.

Alguns autores são de opinião que os pontos separados oferecem ao cirurgião um melhor controle do astigmatismo pós-operatório no que concerne à sua secção, já que podem ser cortados na região do meridiano mais curvo, o que não irá afetar a córnea como um todo ${ }^{(19)}$. Verificando o que ocorreu no presente trabalho, é interessante notar que os altos valores ceratométricos de astigmatismo a favor da regra diminuíram com o tempo. Assim, é importante ter em mente que a secção precoce de algum ponto, pode fazer com que haja uma inversão do tipo de astigmatismo e, nesse caso, o cirurgião deixa de ter controle sobre a situação.

Alguns pacientes apresentam, pré-operatoriamente, astigmatismos corneanos maiores que $2,5 \mathrm{D}^{(20)}$. Para que o cirurgião possa não aumentá-lo ou mesmo reduzi-lo, é necessário que saiba o quanto a técnica cirúrgica utilizada poderá induzir em termos de astigmatismo. Para isso, é necessário mantê-la por um tempo suficiente que lhe permita conhecer seus efeitos em longo prazo, tal como foi feito no presente trabalho.

A cirurgia da catarata oferece a oportunidade de se corrigir, 
pelo menos em parte, a ametropia que o paciente apresentava pré-operatoriamente. Portanto, tão importante quanto o cálculo da lente intra-ocular a ser implantada, é o cuidado que se deve ter no sentido de evitar a indução de astigmatismos indesejáveis ${ }^{(21)}$.

\section{CONCLUSÕES}

1. Considerando a técnica cirúrgica utilizada no presente trabalho, observou-se que os valores de astigmatismo a favor da regra inicialmente verificados tendem a diminuir com o tempo.

2. Observou-se mudança significativa no valor médio do astigmatismo quando foram comparadas as leituras ceratométricas registradas pré-operatoriamente e após o décimo-segundo mês pós-operatório.

\section{ABSTRACT}

Purpose: To study the long-term behavior of induced astigmatism in a series of patients who underwent extracapsular extraction in which a superoposterior limbal incision sutured with interrupted 10-0 mononylon sutures was performed. Methods: 38 eyes of 35 patients were studied, 21 females and 17 males, with ages varying from 47 to 85 year (average $=70.17$ year). All cases were operated by the same surgeon. The postoperative follow-up ranged from 15 to 33 months (average $=22.07$ months). None of the cases had any of the sutures sectioned or removed. All patients recovered a corrected visual acuity equal or superior to 0.5 . To calculate the induced astigmatism in each case, the preoperative and postoperative keratometric readings were considered and a program developed by Jaffe was used. Results: The induced astigmatism ranged from 0.13 diopters $(\mathrm{D})$ to $2.74 \mathrm{D}$ (average $=1.19 \mathrm{D}$ ). Regarding the axis, there was induction of with-the-rule astigmatism in the majority of cases $(60.52 \%)$. A significant shift in the dioptric power of the induced astigmatism was observed when the preoperative keratometric readings were compared with the keratometric readings recorded at the second postoperative week, between the first and the third postoperative months, between the third and the sixth postoperative months and after the twelfth postoperative month. Conclusion: When mononylon 10-0 is used as suture material in extracapsular extraction, the initially observed values of with-the-rule induced astigmatism tend to decrease during the post operative period.

Keywords: Astigmatism/etiology; Cataract extraction; Ophthalmologic surgical procedures/methods; suture techniques; Follow-up studies; Adult

\section{REFERÊNCIAS}

1. Swinger CA. Postoperative astigmatism. Surv Ophthalmol 1987; 31:219-48

2. Maloney WF, Grindle L, Sanders D, Pearcy D. Astigmatism control for the cataract surgeon: a comprehensive review of surgically tailored astigmatism reduction (STAR). J Cataract Refract Surg 1989;15:45-54.

3. Jaffe NS, Jaffe MS, Jaffe GF. Postoperative corneal astigmatism. In: Jaffe NS, Jaffe MS, Jaffe GF, editors. Cataract surgery and special techniques. St. Louis: Mosby; 1997. p.213-29.

4. Terry CM. Surgical control of postoperative astigmatism. In: Engelstein JM ed.- Cataract surgery: current options and problems. Orlando: Grunne \& Stratton; 1984. p.219-23.

5. Albuquerque e Silva, AG. Astigmatismo induzido pela cirurgia de catarata. (Análise de dois grupos nos quais foram realizadas incisões de $15^{\circ}$ e de $120^{\circ}$ a $150^{\circ}$ ). Rev Bras Oftalmol 1982;41:52-62.

6. Reading VM. Astigmatism following cataract surgery. Br J Ophtalmol 1984;68:97-104.

7. Masket S. Comparison of suture materials for closure of the scleral pocket incision. J Cataract Refract Surg 1988;14:548-51.

8. Gorn RA. Surgically induced corneal astigmatism and its spontaneous regression. Ophthalmic Surg 1985;16:162-4.

9. Mendonça CHF, Rezende F. Fixação do globo ocular, retalho conjuntival, incisões e suturas. In: Rezende F, editor. Cirurgia de catarata. Rio de Janeiro: Cultura Médica; 2000. p.85-92.

10. Jaffe NS. Surgical technique. In: Jaffe NS. Cataract surgery and its complications. $3^{\text {rd }}$ ed. St. Louis:Mosby;1981. p.32-91.

11. Ghiaroni A. Influência da técnica de sutura na diminuição do astigmatismo induzido na cirurgia da catarata. Arq Bras Oftalmol 1990;53:259-69.

12. Siegel S. Estadística no parametrica aplicada a las ciencias de la conducta. $2^{\text {nd }}$ ed. México: Trillas; 1975.

13. Cory CC. Prevention and treatment of postimplantation astigmatism. J Cataract Refract Surg 1989;15:58-60.

14. Laflamme MY, Mayer J. L'incision cornéenne inverse dans la chirurgie de la cataracte. Can J Ophtalmol 1985;20:5-8.

15. Jacobi KW, Strobel J. Control of postoperative astigmatism. Trans Ophthalmol Soc UK 1985;104:715-26.

16. Gills JP. My method of extracapsular cataract extraction with implantation of a posterior chamber intraocular lens. Ophthalmic Surg 1985;16:386-92.

17. Drews RC. Five year study of astigmatic stability after cataract surgery with intraocular lens implantation: comparison of wound sizes. J Cataract Refract Surg 2000;26:250-3.

18. Richards SC, Brodstein RS, Richards WL, Olson RJ, Combe PH, Crowll KE. Long-term course of surgically induced astigmatism. J Cataract Refract Surg 1988;14:270-6.

19. Brown NA, Sparrow JM. Control of astigmatism in cataract surgery. Br J Ophthalmol 1988;72:487-93.

20. Bartholomew RS. Post-cataract astigmatism: its control and correction. Aust NZJ Ophthalmol 1988;16:215-27.

21. Ghiaroni A. Catarata e astigmatismo. In: Rezende F, editor. Cirurgia da catarata. Rio de Janeiro: Cultura Médica; 2000. p.381-12.

\section{Nos artigos enviados para publicação, o nome dos autores}

e suas afiliações devem estar completos. Isso facilitará a indexação e os links com as bases de dados e o CV Lates. 